Original Research Paper

\title{
On Semicontinuous Nonclassical Ordinary Differential Inclusions with Nonlocal Condition
}

\author{
${ }^{1}$ Sheila Amina Bishop, ${ }^{2}$ Godwin Amechi Okeke and ${ }^{1}$ Timothy Ashibel Anake \\ ${ }^{\text {I} D e p a r t m e n t ~ o f ~ M a t h e m a t i c s, ~ C o v e n a n t ~ U n i v e r s i t y, ~ O t a, ~ P . M . B . ~ 1023, ~ O g u n ~ S a t e, ~ N i g e r i a ~}$ \\ ${ }^{2}$ Department of Mathematics, \\ Michael Okpara University of Agriculture, Umudike, P.M.B. 7267, Umuahia, Abia State, Nigeria
}

Article history

Received: 08-03-2017

Revised: 26-03-2017

Accepted: 11-05-2017

Corresponding Author:

Sheila Amina Bishop

Department of Mathematics,

Covenant University, Ota,

Ogun Sate, Nigeria

Email:

sheila.bishop@covenantunivers

ity.edu.ng

\begin{abstract}
In this study, we examined existence of lower semicontinuous solution of Nonclassical Ordinary Differential Inclusions (QSDIs) with Nonlocal Conditions. Here the multivalued stochastic processes are lower semicontinuous.
\end{abstract}

Keywords: Nonlocal Conditions, Lower Semicontinuous, Multivalued Stochastic Processes, Fixed Point

\section{Introduction}

In recent times, several researchers have carried out studies on differential inclusions with lots of emerging results such as semilinear evolution inclusions with nonlocal conditions and upper semicontinuous multivalued maps. See (Aitalioubrahim, 2011; Boucherif, 2009; Gatsori et al., 2004; Zhu and Li, 2008). (Cardinali et al., 2008; Cardinali and Rubbioni, 2012), established some results on local mild solutions and impulsive mild solutions for semilinear inclusions. Aitalioubrahim (2011) established some new results on mild solution of semilinear noncovex differential inclusion. The following were considered: the case when the set-valued map is a non-open multifunction with measurability and Lipschitz continuity conditions imposed on the first and second variables respectively. These recent studies have shown that differential inclusions and problems with nonlocal conditions are of more practical applications in real life when compared to problems with local conditions (Antosiewicz and Cellina, 1975; Bishop et al., 2016; Cellina, 1988).

Within the setting of quantum stochastic calculus Ekhaguere (1992), not much has been done. However, Ayoola (2008), Bishop and Ayoola (2015), studied the topological properties of solution sets for Lipschitz and non Lipschitz Quantum Stochastic Differential Inclusions (QSDIs) under the local conditions with the multivalued stochastic processes been continuous. Ogundiran and Payne (2014) considered a unified treatment of existence of solution of both upper and lower semicontinuous quantum stochastic differential inclusions under the local condition. Bishop et al. (2016) established some new results on impulsive nonclassical ordinary differential equations. The initial conditions are not necessarily local and the multivalued stochastic processes are lower semicontinuous. Problems with lower semicontinuous maps have more application especially when dealing with dynamical systems.

In this study, we present new results on QSDIs with nonlocal conditions where the multivalued stochastic processes are lower semicontinuous.

This problem will have practical applications in the theory of quantum dynamical systems.

The result of this paper generalizes some of the results of Bishop et al. (2016) and also extends the work of Aitalioubrahim (2011) to the class of noncummutative quantum setting.

We consider the following lower semicontinuous quantum stochastic evolution inclusion:

$$
\begin{aligned}
& d x(t) \in A(t) x(t)+\left(E(t, x(t)) d \Lambda_{\pi}(t)+F(t, x(t)) d A_{g}(t)\right. \\
& \left.+G(t, x(t)) d A_{f}^{+}(t)+H(t, x(t)) d t\right), \\
& x(0)=g(x(.)), \quad t \in[0, T] \subseteq \mathbb{R}_{+}
\end{aligned}
$$

The term in the bracket on the right hand of Inclusion 1 is the formulation of Hudson and Parthasarathy (1984) Boson quantum stochastic calculus. E, F, G, H are 
coefficients that lie in the space $L_{l o c}^{2}([0, T] \times \tilde{\mathcal{A}})_{m v s}$, the space $\tilde{\mathcal{A}}$ is necessarily locally convex, $g: C([0, T], \tilde{\mathcal{A}}) \rightarrow \tilde{\mathcal{A}}, A$ is a family of densely defined linear operator. However, it has been shown by Ekhaguere (2007) that the following evolution inclusion:

$$
\begin{aligned}
& \frac{d}{d t}\langle\eta, x(t) \xi\rangle \in A(t) x(t)+P(t, x)(\eta, \xi) \\
& x(0)=g(x(.)), t \in[0, T]
\end{aligned}
$$

is equivalent to Inclusion 1, where the map $(t, x) \rightarrow P(t, x)(\eta, \xi)$ in Inclusion 2 is a multivalued sesquilinear form on $(\mathbb{D} \otimes \mathbb{E})^{2}$ with its explicit form defined by Ekhaguere (2007). We organize the rest of this paper as follows: Section 2, will consist of preliminaries while in Section 3, the main result will be considered.

\section{Notations and Preliminary Results}

In this section we shall adopt the fundamental concepts and structures as in the references (Ayoola, 2004; Ekhaguere, 2007). We employ the space $\tilde{A}$ of noncommutative stochastic processes whose topology $\tau_{w}$ is generated by the family of seminorms $\left\{\left\|x_{\eta \xi}\right\|=|\langle\eta, x \xi\rangle|, x \in \tilde{A}, \eta, \xi \in(\mathbb{D} \otimes \mathbb{E})\right\}$. The elements of $\tilde{A}$ consists of linear maps from $\mathbb{D} \otimes \mathbb{E}$ into $\mathfrak{R} \otimes \Gamma\left(L_{\gamma}^{2}\left(\mathbb{R}_{+}\right)\right)$having domains of their adjoints containing $\mathbb{D} \otimes \mathbb{E}_{\infty}$. In what follows, as in (Bishop and Ayoola, 2015; Ekhaguere, 1992; 2007; Ogundiran and Payne 2014) we employ the definitions and notations of the spaces $L_{\gamma, l o c}^{P}(\tilde{A}), L_{l o c}^{\infty}\left(\mathbb{R}_{+}\right), \quad L_{\text {loc }}^{2}([0, T] \times \tilde{A})_{m v s}, \gamma . \mathbb{D}$ is some pre-Hilbert space with $\mathfrak{R}$ as its completion. Let $S$ be a topological space, then $\operatorname{clos}(S)$, denotes the collection of all nonempty closed subsets of $S$ while $\operatorname{Comp}(S)$ denotes the collection of all nonempty compact subsets of $S$. We shall employ the Hausdorff topology on $\operatorname{clos}(\tilde{A})$. By Theorem V.5 of Reed and Simon (1980), the $\sigma$-weak topology $\tau_{\sigma w}$ is metrizable since $\mathbb{D} \otimes \mathbb{E}_{\infty}$ has a countable base, hence $\tilde{A}$ is metrizable. For more on Banach space, metrizable spaces, etc., see Krein (1971).

\section{Definitions 1:}

- A multivalued stochastic process $\Phi$ with values in $\operatorname{clos}(\tilde{A})$, with $I \subseteq \mathbb{R}_{+}$as its basis, is a multivalued function on the interval $I$

- If the above holds, then a selection of $\Phi$ is a stochastic process $x: I \rightarrow \tilde{A}$ such that $x(t) \in \Phi(t)$ for almost all $t \in I$
Note: All through the remaining part of this paper, $\Phi$ is multivalued stochastic process except stated otherwise.

- $\Phi$ is adapted if $\Phi(\mathrm{t}) \subseteq \tilde{\mathcal{A}}_{t}, t \in \mathbb{R}_{+}$

- $\Phi$ is computable if $t \rightarrow d_{\eta \xi}(x, \Phi(t))$ is computable, $\eta, \xi \in(\mathbb{D} \otimes \mathbb{E}), x \in \tilde{A}$

- $\Phi$ is locally absolutely p-integrable if $t \rightarrow \Phi(t)_{\eta \xi} \in$ $L_{\text {loc }}^{P}(I), t \in \mathbb{R}_{+} \quad \eta, \xi \in(\mathbb{D} \otimes \mathbb{E})$. This will be denoted by $L_{l o c}^{P}(\tilde{A})_{m v s}$

\section{Notations 1:}

- $\quad L_{l o c}^{P}(I \times \tilde{A})_{m v s}$ is the set of maps $\Phi: I \times \tilde{A} \rightarrow \tilde{A}$ such that $t \rightarrow \Phi(t, \quad x(t)) \quad \in L_{l o c}^{P}(\tilde{A})_{m v s} \forall \quad x \in L_{l o c}^{P}(\tilde{A})_{m v s}$, $P \in(0, \infty), I \subseteq \mathbb{R}_{+}$

- For the purpose of this work, $f, g \in L_{\gamma}^{\infty}\left(\mathbb{R}_{+}\right), \pi \in L_{B(\gamma)}^{\infty}\left(\mathbb{R}_{+}\right)$, while $\Lambda_{\pi}, A_{f}$ and $A_{g}^{+}$are stochastic processes

\section{Definition 2:}

- Let $C(I, \tilde{A})$, denote the space of all stochastic processes that are continuous and $L^{1}(I, \tilde{A})$ denotes the space of all stochastic processes that are $L^{1}$ measurable or $L^{1}$-computable. We define the sesquilinear equivalent form $C(I, \operatorname{ses} q(\mathbb{D} \unrhd \mathbb{E})), L^{1}(I$, $\operatorname{ses} q(D \times E))$, in the same manner with $x: I \rightarrow \operatorname{ses} q(\mathbb{D} \otimes \mathbb{E})$. The Banach space $C(I, \operatorname{ses} q(\mathbb{D} \otimes \mathbb{E}))$ is equipped with the norm $\|x\|_{\eta \xi, c}=\sup \{|x(t)(\eta, \xi)|, t \in I\}$

- $E \subset L^{1}(I, \tilde{A})$ is decomposable if for all measurable (computable) $u(),. v(.) \in E$, the function $u(.)_{\chi} I(.)_{\chi}+$ $v(.)_{\chi} I(.)_{\chi} \in E, \quad I \subseteq[0, T]$, where $\chi I($.$) denotes the$ characteristic function

- A multivalued stochastic process $\Phi: I \times \tilde{\mathcal{A}} \rightarrow 2^{\tilde{\mathcal{A}}}$ is lower semicontinuous (1.s.c.) if $\forall$ open set $V \subset \tilde{A}$, $\Phi^{-}(V)$ is open

- $\Phi$ is completely continuous if $\Phi(E)$ is relatively compact for every $E$ bounded set of $D$, where $D$ is a non empty subset of $\tilde{A}$

- Assume that the multivalued map $P:[0, T] \times \tilde{A} \rightarrow 2^{\tilde{A}}$ has nonempty compact values and $\mathcal{F}$ is the Niemytzki operator. Define the operator $\mathcal{F}: C([0, T], \tilde{A}) \rightarrow 2^{L^{\prime}([0, T], \tilde{A})}$ by $\mathcal{F}(x().(\eta, \xi))=\{y(.) \in$ $\left.\left.L^{1}([0, T]), \tilde{A}\right): y_{\eta \xi}(t) \in P(t, x(t))(\eta, \xi), t \in[0, T]\right\}$ 
The following result established by Ayoola (2008) will be useful in establishing the main result. The proofs are simple adaptation of arguments employed in the reference.

\section{Lemma 1}

Let $\tilde{A}$ be a metrizable space and let $\Phi: I \times \tilde{A} \rightarrow 2^{L^{\prime}([0, T], \tilde{A})}$ be a multivalued operator which is lower semicontinuous (1.s.c) and has nonempty closed and decomposable values. Then $\Phi$ has a continuous selection. That is there exists a continuous function $P$ : $\tilde{A} \rightarrow L^{1}([0, T], \tilde{A})$ such that $P(y) \in \Phi(y) \forall y \in \tilde{A}$.

\section{Main Results}

Here, we examine the existence of mild solution of an evolution inclusion where the multivalued map is lower semicontinuous. We consider the nonlocal QSDI with nonlocal conditions:

$$
\begin{aligned}
& \frac{d}{d t} \eta, x(t) \xi \in A(t) x(t)+P(t, x)(\eta, \xi) \\
& x(0)=g(x(.)), t \in[0, T]
\end{aligned}
$$

Let $\{A(t): t \in[0, T]\}$ be defined on $\tilde{A}$ and $g: C([0, T], \tilde{A})$ $\rightarrow \tilde{A}$. Define an evolution operator $T(.,$.$) by \mathrm{T}: \Delta=\{(t, s)$ : $0 \leq s \leq t \leq T\} \rightarrow \tilde{A}, A(t)$ generates $T(.,$.$) . As outlined by$ Aitalioubrahim (2011) the operators $T(t, s)$ are strongly differentiable such that $T(t, r) T(r, s)=T(t, s) \forall 0 \leq s \leq r \leq$ $b$, while $\frac{\partial T(t, s)}{\partial t}=A(t) T(t, s)$ and $\frac{\partial T(t, s)}{\partial s}=-A(t) T(t, s) \forall$ $(t, s) \in \Delta$. For more on the operators $T(t, s)$ see Aitalioubrahim (2011) and the references therein.

\section{Definition 3}

Assume that E, F, G, $\mathrm{H} \in L_{l o c}^{2}(I \times \tilde{\mathcal{A}})_{m v s},\left(t_{0}, x_{0}\right)$ a fixed point of $I \times \tilde{A}$. Then the following Equation:

$$
\eta, \varphi(t) \xi=\eta, T(t, s) g(\varphi) \xi+\int_{0}^{t} T(t, s) P(s, \varphi(s))(\eta, \xi) d s
$$

will be called a solution of Equation 1 respectively Equation 2 for arbitrary $\eta, \xi \in(\mathbb{D} \otimes \mathbb{E})$.

\section{Theorem 1}

Assume that the function $g: \tilde{A} \rightarrow C([0, T], \tilde{A})$ is continuous and the map $P: I \times \tilde{A} \rightarrow L^{\tilde{A}}$ has non-empty compact values. Then:

- $\quad\left(S_{i}\right)(t, s) \rightarrow P(t, x)(\eta, \xi)$ is $\mathrm{L}^{1}$-measurable

- $\quad\left(S_{i i}\right) t \rightarrow P(t, x)(\eta, \xi)$ is 1.s.c for almost all $t \in[0, T]$

- $\left(S_{i i i}\right) M(.) \in L^{1}\left([0, T], \mathbb{R}_{+}\right)$is a function such that:

$$
|P(t, x)(\eta, \xi)|:=\sup \left\{\|y\|_{\eta \xi}: y \in\langle\eta, P(t, x) \xi\rangle\right\} \leq M(t)
$$

for almost all $t \in[0, T], x \in \tilde{A}$.

- $\quad\left(\mathrm{S}_{\mathrm{iv}}\right)$ Let $N>0$ and $|T(t, s)| \leq N$ for each $(t, s) \in \Delta$

- $\quad\left(S_{v}\right)$ Let $m$ and $l$ be non-negative constants, then

$$
\|g(x)\|_{\eta \xi} \leq m\|x(.)\|_{\eta \xi}+l \forall x(t) \in C([0, t], \tilde{\mathcal{A}})
$$

- $\left(S_{v i}\right)$ Let $B \subseteq C(I, \tilde{A}), t \in I$ and $\sup _{t \in I}\|x(t)\|_{\eta \xi}<\infty$, then, the set:

$$
\left\{T(t, 0) g(y(.))+\int_{0}^{t} T(t, s) \mathcal{P}(y(.))(s) d s, y \in B\right\}
$$

is relatively compact in $\tilde{A} . P: C([0, \mathrm{t}], \tilde{A}) \rightarrow L^{1}([0, \mathrm{t}]$, $\tilde{A})$ is such that $P(y().) \in F(y().) \forall y \in C([0, t], \tilde{A})$. Then if $N m<1$, Inclusion 1 equivalently Inclusion 2 has at least one mild solution on the given interval.

\section{Proof}

From $\left(S_{i i}\right)$, P is 1.s.c and by Lemma $1, P(y().) \in F(y()$. $\forall y \in C([0, t], \tilde{A})$. Considering the problem:

$\frac{d}{d t}\langle\eta, y(t) \xi\rangle \in A(t) y(t)+\mathcal{P}(y()).(\eta, \xi)$

$y(0)=g(y()),. \quad t \in[0, T]$

By implication, if $y(.) \in C([0, t], \tilde{A})$ is a solution of Inclusion 5, then $y($.$) is also a solution of Inclusion 2$. Next, we transform Inclusion 5 into a fixed point form:

$\forall \quad y(.) \in C([0, t], \tilde{A}), \quad$ again define $\mathcal{P}: C([0, T], \operatorname{ses} q(\mathbb{D} \otimes \mathbb{E})) \rightarrow C([0, T], \operatorname{ses} q(\mathbb{D} \otimes \mathbb{E}))$ by:

$$
\begin{aligned}
& \gamma(y(.))(t)(\eta, \xi)=T(t, 0) g(y(.))(\eta, \xi) \\
& +\int_{0}^{t} T(t, s) \mathcal{P}(y(.))(s)(\eta, \xi) d s
\end{aligned}
$$

The proof is presented as follows:

- Show that $\gamma$ is continuous

- Show that $\gamma$ is bounded on bounded sets of $C([0, T], \operatorname{ses} q(\mathbb{D} \otimes \mathbb{E}))$. We do this by showing that $\chi\left(B_{r}\right)$ is bounded $\forall r \geq 0, \quad B_{r}=\left\{y_{\eta \xi}(.) \in\right.$ $\left.C([0, T], \operatorname{ses} q(\mathbb{D} \otimes \mathbb{E})):\|y(.)\|_{\eta \xi} \leq r\right\}$

- Show that $\gamma$ maps bounded sets into sets that are equicontinuous

- Lastly we show that for some $0<\gamma<1$ the set $R(y)=\{y(.) \in C([0, T], \operatorname{ses} q(\mathbb{D} \mathbb{\otimes} \mathbb{E})): \gamma y()=.\gamma(y())$. is bounded

Let $\left(y_{\eta \xi, k}\right)_{k \geq 0}$ converge to $y_{\eta \xi}$ in $C([0, T]$,sesq $(\mathbb{D} \otimes \mathbb{E}))$. 
Then $\forall t \in[0, T]$ :

$\left\|\Phi\left(y_{k}(.)\right)(t)-\Phi(y(.))(t)\right\|_{\eta \xi} \leq|T(t, s)|\left\|\mathrm{g}\left(y_{k}(.)\right)-\mathrm{g}(y(.))\right\|_{\eta \xi}$

$+\int_{0}^{t}\left|T(t, s) \| \mathcal{P}\left(y_{k}().\right)(s)(\eta, \xi)-\mathcal{P}(y()).(s)(\eta, \xi)\right| d s$

$\leq N\left\|\mathrm{~g}\left(y_{k}(.)\right)-\mathrm{g}(y(.))\right\|_{\eta \xi}$

$+N \int_{0}^{t}\left|T(t, s) \| \mathcal{P}\left(y_{k}().\right)(s)(\eta, \xi)-\mathcal{P}(y()).(s)(\eta, \xi)\right| d s$

Since $g\left(y_{k}().\right) \rightarrow g(y()$.$) as k \rightarrow \infty$ and $P$ is continuous, we deduce that $\Phi$ is continuous.

Let $h_{\eta \xi} \in \Phi\left(B_{r}\right)$, we have $\forall t \in[0, T]$ :

$$
\begin{aligned}
& \left|h_{\eta \xi}(t)\right| \leq|T(t, 0)|\|g(y(.))\|_{\eta \xi}+\int_{0}^{t}|T(t, s) \| \mathcal{P}(y(.))(s)(\eta, \xi)| d s \\
& \leq N\left[m\|y(.)\|_{\eta \xi}+l \int_{0}^{T} M(s) d s\right] \\
& \leq N\left[(m r+l)+\int_{0}^{T} M(s) d s\right] \leq N \lambda
\end{aligned}
$$

Therefore, $\Phi\left(B_{r}\right) \subset B_{N \lambda}$, where $\left[(m r+l)+\int_{0}^{T} M(s) d s\right]=\lambda$.

Since $h_{\eta \xi} \in \Phi\left(B_{r}\right)$ and $y(.) \in B_{r}$, then $h_{\eta \xi} \in \Phi\left(y_{\eta \xi}().\right)$ and we have for $t<s, t \in[0, T]$ :

$$
\begin{aligned}
& \left|h_{\eta \xi}(t)\right| \leq|T(s, 0)-T(t, 0)|\|g(y(.))\|_{\eta \xi} \\
& +\int_{t}^{s} T(s, \tau) \| \mathcal{P}(y(.))(\tau)(\eta, \xi) \mid d \tau \\
& +\int_{0}^{t}|T(t, \tau)-T(s, \tau) \| \mathcal{P}(y(.))(\tau)(\eta, \xi)| d \tau \\
& \leq N(m r+l)|T(s, 0)-T(t, 0)|+\int_{t}^{s} M(\tau) d \tau \\
& +N\left(+\int_{t}^{T}|T(s, 0)-T(t, 0)| M(\tau) d \tau\right) \rightarrow 0
\end{aligned}
$$

as $s \rightarrow t$.

By the property of $T(t, s), t>s$ and the fact that it is a strongly continuous operator, we have by Arzela Ascoli theorem the convergence in the uniform operator topology.

Let $y(.) \in R(y)$, then we have:

$$
\begin{aligned}
& y(.)(\eta, \xi)=\gamma[T(t, 0) g(y(.))(\eta, \xi) \\
& \left.+\int_{0}^{t} T(t, s) \mathcal{P}(y(.))(\tau)(\eta, \xi)\right] d s \\
& \leq \gamma N\left(m\|y(.)\|_{\eta \xi}^{\infty}+l\right)+\gamma N \int_{0}^{T} M(s) d s
\end{aligned}
$$

and:

$$
\begin{aligned}
& \|y(.)\|_{\eta \xi}-\gamma N m\|y(.)\|_{\eta \xi}=\|y(.)\|_{\eta \xi}(1-\gamma N m) \\
& \leq \gamma N\left(l+\int_{0}^{T} M(s) d s\right)
\end{aligned}
$$

Since $\quad 1-\gamma N m>1-N m$, hence, we get $\|y(.)\|_{\eta \xi}^{\infty} \leq \frac{\gamma N\left(l+\int_{0}^{T} M(s) d s\right)}{1-\gamma N m}$.

This shows that $R(y)$ is bounded. Consequently $\|y(.)\|_{\eta \xi}^{\infty} \leq v$ for some constant $v$ and by Arzela Ascoli theorem, $\Phi$ is completely continuous.

Hence by Schaefer's theorem, the conclusion is that $\Phi$ has a fixed point and this fixed point is a mild solution of Equation 5.

\section{Conclusion}

Results obtained here generalize analogous results due to the references (Aitalioubrahim, 2011; Cardinali et al., 2008; Cardinali and Rubbioni, 2012) which concern classical ordinary differential equations and some of the results of Bishop et al. (2016). Hence this will be an extension of the results on Quantum Stochastic Differential Inclusion (QSDI) in the literature.

\section{Acknowledgement}

The authors are grateful to the management of Covenant University for their research support grant. They will also want to appreciate the reviewer for the useful criticism and corrections.

\section{Authors' Contributions}

All authors have made remarkable contributions in this research work.

\section{Conflict of Interest}

The authors declare that there is no conflict of interest regarding the publication of this manuscript.

\section{References}

Aitalioubrahim, M., 2011. Existence of mild solutions of a semilinear nonconvex differential inclusion with nonlocal conditions. Matematnykn Bechnk, 63: 181-190.

Antosiewicz, H.A. and A. Cellina, 1975. Continuous selections and differential relations. J. Differ. Eq., 19: 386-398.

DOI: $10.1016 / 0022-0396(75) 90011-\mathrm{X}$

Ayoola, E.O., 2008. Topological properties of solution sets of Lipschitzian quantum stochastic differential inclusions. Acta Applic. Math., 100: 15-37. DOI: $10.1007 / \mathrm{s} 10440-007-9175-1$

Ayoola, E.O., 2004. Continuous selections of solution sets of lipschitzian quantum stochastic differential inclusions. Int. J. Theor. Phys., 43: 2041-2058. DOI: 10.1023/B:IJTP.0000049009.71965.c0 
Bishop, S.A., E.O. Ayoola and G.J. Oghonyon, 2016. Existence of mild solution of impulsive quantum stochastic differential equation with nonlocal conditions. Anal. Math. Phys., 6: 109-210. DOI: $10.1007 / \mathrm{s} 13324-016-0140-\mathrm{x}$

Bishop, S.A. and E.O. Ayoola, 2015. On topological properties of solution sets of non Lipschitzian quantum stochastic differential inclusions. Anal. Math. Phys., 5: 217-320.

DOI: $10.1007 / \mathrm{s} 13324-015-0109-1$

Boucherif, A., 2009. Semilinear evolution inclusions with nonlocal conditions. Applied Math. Lett., 22: 1145-1149. DOI: 10.1016/j.aml.2008.10.004

Cardinali, T., F. Portigiani and P. Rubbioni, 2008. Local mild solutions and impulsive mild solutions for semilinear Cauchy problems involving lower Scorza-Dragoni multifunctions. Topol. Meth. Nonlinear Anal., 32: 247-259.

Cardinali, T. and P. Rubbioni, 2012. Mild solutions for semilinear differential inclusions with nonlocal conditions in Banach spaces. Nonlinear Anal., 75: 871-879.

Cellina, A., 1988. On the set of solutions to Lipschitzian differential inclusions. Diff. Integral Eq., 1: 495-500.

Ekhaguere, G.O.S., 1992. Lipschitzian quantum stochastic differential inclusions. Int. J. Theor. Phys., 31: 2003-2034. DOI: 10.1007/BF00671969
Ekhaguere, G.O.S., 2007. Topological solutions of noncommutative stochastic differential equations. Stoch. Anal. Applic., 25: 961-993. DOI: $10.1080 / 07362990701540345$

Gatsori, E., S.K. Ntouyas and Y.G. Sficas, 2004. On a nonlocal Cauchy problem for differential inclusions. Abstract Applied Anal., 5: 425-434. DOI: $10.1155 / \mathrm{S} 108533750430610 \mathrm{X}$

Hudson, R.L. and K.R. Parthasarathy, 1984. Quantum Ito's formula and stochastic evolutions. Comm. Math. Phys., 93: 301-324. DOI: $10.1007 / \mathrm{BF} 01258530$

Krein, S.G., 1971. Linear Differential Equations in Banach Space. 1st Edn., American Mathematical Society, Providence, ISBN-10: 0821886584, pp: 390.

Ogundiran, M.O. and V.F. Payne, 2014. On solution sets of semicontinuous quantum stochastic differential inclusions. Applied Math. E-Notes, 14: 135-143.

Reed, M. and B. Simon, 1980. Methods of Modern Mathematical Physics: Functional Analysis. 1st Edn., Academic Press, Inc., New York, ISBN-10: 0125850506, pp: 400.

Zhu, L. and G. Li, 2008. On a nonlocal problem for semilinear differential equations with upper semicontinuous nonlinearities in general Banach spaces. J. Math. Anal. Applic., 341: 660-675. DOI: $10.1016 /$ j.jmaa.2007.10.041 